\title{
Knowledge, Attitude, and Practice Regarding COVID-19 among Physicians in Egypt
}

\author{
Almass F. Taie', Tayseer M. Metwally ${ }^{2 *}$, Sarah S. Mahdy ${ }^{1}$, and Heba M. Abbas ${ }^{1}$ \\ 'Department of Family Medicine, Faculty of Medicine, Suez Canal University, Ismailia, and ${ }^{2}$ Department of Family \\ Medicine, Faculty of Medicine, Suez University, Suez, Egypt.
}

\begin{abstract}
Background: Protection of health care workers and prevention of intra-hospital transmission of infection are important aspects in COVID-19 epidemic response, and this requires that they must have updated knowledge regarding the source, transmission, symptoms, and preventive measures. Lack of knowledge and misunderstandings leads to delayed diagnosis, spread of disease, and poor infection control practice. Objective: To identify the status of knowledge, attitude, and practice regarding COVID-19 among physicians in Egypt. Subjects and Methods: A descriptive cross-sectional survey-based study was carried out on 160 physicians who participated in the study which was conducted in May and June 2020. Self-administered questionnaire shared on social media groups of Egyptian physicians to assess knowledge, attitude, and practice among physicians regarding COVID-19. Results: Participants have good knowledge (98.8\%), a positive attitude means $9.76 \pm 1.70$, and good practice (77.5\%). physicians working in clinical departments had greater knowledge than physicians working in academic departments $(p=0.003)$ also physicians with work experience $\geq 5$ years have greater knowledge $(p=0.002) .88 .75 \%$ of physicians perceived that limited infection control material and $84.37 \%$ of them perceived that Lack of policy and procedures of infection control practice were the major barriers to infection control. Conclusion: There is good knowledge regarding COVID-19 among Egyptian physicians. Appropriate practice correlates with better knowledge and a positive attitude towards COVID-19 infection. More training programs on protective measures to improve attitude among physicians and healthcare workers are necessary to win the fight against COVID-19 infection.
\end{abstract}

Keywords: Attitude; COVID-19; Egypt; Knowledge; Practice

\section{Introduction}

Coronaviruses are a large group of viruses that are rather common throughout the community. Historically, evidence has shown that the virus is transmitted through birds and mammals, with humans being particularly vulnerable to infection and transmission of the virus. The previous outbreaks of coronaviruses such as severe acute respiratory syndrome. Coronavirus (SARS-CoV) and Middle East Respiratory Syndrome-Corona virus (MERS-CoV) in 2003 and 2015 show similarities to the novel coronavirus, which was first reported in December 2019, and is currently the disease in questions resulting originated in Wuhan, the capital city of Province Hubei, China, and on 31 December 2019, with the emergence of more such cases, 
Wuhan gained attention by World Health Organization $(\mathrm{WHO})^{(2)}$. The pathogen identified named as novel coronavirus (2019nCoV), currently called severe acute respiratory syndrome corona virus-2 (SARS-CoV2), an enveloped and single-stranded RNA virus(3), which has a phylogenetic resemblance to SARS-COV-1(4). Owing to rapid spread of this deadly virus from the epicenter to number of countries, WHO declared it as public health emergency of international concern (PHEIC) on January 30, $2020^{(5)}$. Later, due to uncased fast spread, the severity of illness, and the continual escalation in number of affected countries, cases, and causalities, WHO declared coronavirus disease 2019 (COVID-19) a global pandemic on 11 March 2020. To date (8 Dec 2020), the COVID-19 have spread to more than 215 countries and territories accounted for $68,232,114$ laboratory-confirmed cases and 1,556,552 mortalities also attributed to this deadly pathogen ${ }^{(5)}$. Regarding Egypt to date (8 Dec 2020) there are 118,847 laboratory confirmed cases and 6,790 deaths that make Egypt at the $62^{\text {nd }}$ order Out of 215 regions and countries around the world. The death rate of the total number of injured (6, 04\%) After Romania and Andorra in Europe (6.1\%), Egypt is preceded by Switzerland $(6,01 \%)$ and the United States of America (5.9\%). Nicaragua in Central America comes first (31.3\%) It was followed by the latter of MS Zandam (22.2\%), Yemen (20\%), and Saint Martin in North America (18.4\%), compared to all countries and regions in the world(6). COVID-19 transmits from person to person by droplets when an infected person sneezes and by direct contact and virus has an incubation period of 4-14 days. Elderly and patients who suffered from chronic medical conditions like diabetes and cardiovascular diseases are more likely to get severe infections. The main manifestations of COVID-19 at the time of writing this manuscript are fever, dry cough, dyspnea, myalgia, fatigue, lymphopenia, and radiographic evidence of pneumonia. Complications (e.g., acute respiratory distress syndrome [ARDS], arrhythmia, shock, acute cardiac injury, secondary infection, and acute kidney injury) and death may occur in severe $\operatorname{cases}^{(7)}$. Presently, no antiviral therapy or vaccine is explicitly recommended for COVID-19, and the implementation of preventive meas-ures to control COVID-19 is the mainstay of critical intervention. In terms of symptoms, more than $80 \%$ of COVID-19 patients showed mild symptoms and recovered without any medical intervention, approximately $20 \%$ of infected cases had a severe illness such as shortness of breath, septic shock, and multi-organ failure, and it has been reported that an estimated $2 \%$ of cases can be fatal. The risk of increased severity was noticed in the elderly and with underlying chronic diseases $^{(8)}$. Healthcare professionals (HCPs) of all levels and kinds are primarily involved in catering to patients of this highly transmittable pathogen. COVID-19 has posed a serio to the HCP owing to their frequent exposure to infected individuals ${ }^{(9)}$. Protection of HCPs and prevention of intra-hospital transmission of infection are important aspects in epidemic response and this requires that HCPs must have updated knowledge regarding the source, transmission, symptoms, and preventive measures ${ }^{(10)}$. Literature suggests that lack of knowledge and misunderstandings among HCPs leads to delayed diagnosis, spread of disease, and poor infection control practice ${ }^{(11)}$. Several thousand healthcare workers have already been infected, mainly in China; preventing intrahospital transmission of communicable disease is, therefore, a priority ${ }^{(9)}$. Although educational campaigns have increased their awareness regarding COVID-19 yet it remains unclear to what extent this 
knowledge can be put into practice. Knowledge, attitude, and practice survey provide a suitable format to evaluate existing programs and to identify effective strategies for behavior change in society. Currently, there is scarce information regarding the awareness level of physicians in Egypt, Therefore, the present study aimed to identify the current status of knowledge, attitude, and practices regarding COVID-19 among physicians in Egypt and then improve it to decrease the incidence between physicians.

\section{Subjects and Methods}

A descriptive cross-sectional survey-based study was conducted in May and June 2020 as it was not feasible to conduct a population-based survey in this critical condition; the investigators selected an online data collection method. The sample size was calculated using Epi info 7 using the prevalence of knowledge and practice (93.2 and $88.7 \%$ respectively $)^{(6)}$ with confidence level $95 \%$ and margin of error $5 \%$ and study population assumed to be 80,000 . The highest sample size obtained was 154 participants. A convenient sample was collected till the required sample size was achieved on 15th June 2020. Egyptian physicians with different specialties and different scientific degrees were eligible for participation in this survey after their agreement. A questionnaire was designed on Google forms and link generated was shared on social media groups of physicians. Link also was shared personally to physicians who are in contact list of investigators. We used a reliable and valid questionnaire after approval of Saqlain $M$ et $\mathrm{al}^{(6)}$, it was and consisted of questions assessing demographics, information source, knowledge, attitude, practice toward, COVID-19 and perceived barriers in infection control practice (appendix 1). Demographic characteristics included are gender, age, experience, and specialty, and one item regarding source of information about COVID-19. Knowledge section consisted of 14 items: regarding nature of disease, etiology, symptoms, risk group, testing, transmission, treatment, and precautions / preventions. Each question was responded as yes, No and I don't know. The correct answer was marked as 1 while the wrong answer was marked as 0 . The total score ranged from $0-14$ and a cutoff level of $\leq 10$ was set for poor knowledge and $\geq 11$ ( $>75 \%$ ) for good knowledge. The attitude section consisted of 7 items assessing the attitude of healthcare workers toward treatment, infection control procedure, and information regarding COVID-19. Response of each item was recorded on a 5-point Likert scale as follows strongly agree (1-point), agree (2-point), Undecided (3-point), disagree (4-point), and strongly disagree (5-point). The total score ranged from 7 to 35, with an overall lower mean score indicating a positive attitude toward COVID-19. The practice section included 6 items regarding the use of face masks, and the practice of other precautionary measures. Each item was responded as yes (1-point), No (o-point), and sometimes (0point). Practice items total score ranged as $0-6$, and a score of $\geq 4$ demonstrates good practice, and a score of $<4$ indicated poor practice toward precautionary measures of COVID-19. Eight items to assess the perception of HCPs regarding barriers in infection control practice. Each question was respondent to on 5-point Likert scale as follows strongly agree, agree, undecided, disagree, and strongly disagree. Responses were presented as frequencies and percentages.

\section{Ethical Considerations}

All the procedures of this study were approved by the Suez Canal University Hospital and the family medicine department. 
Approval from the research Ethics Committee, faculty of medicine Suez Canal University was taken before starting data collection. The study questionnaire contained a consent portion that data is confidential, and participation is voluntary. All procedures performed in the study were following the ethical standards of the institutional research committee of the Faculty of Medicine Suez Canal University and with the 1964 Helsinki declaration and its later amendments. Informed consent was obtained from all individual participants included in the study. Data Available on request from the corresponding author

\section{Statistical analysis}

Data was entered in SPSS V.21 for statistical analysis. Numerical variables were measured as mean and standard deviations while categorical variables were expressed as frequencies and percentages. Chisquare tests were applied to find a difference in knowledge groups (good vs poor) and practice (good vs poor) by demographic characteristics. Independent sample t-test analysis was performed in assessing any difference in mean attitude score by demographic characteristics. Pearson-rank correlation test was applied to find any correlation between knowledge, attitude, and practice sections. $p$-value of less than 0.05 was considered as significant in all tests.

\section{Results}

Of the 160 participating physicians $67.5 \%$ were between 30 and 40 years old, most of the participants $71.9 \%$ were females. Of the respondents, $86.2 \%$ had work experience of more than 5 years, $88.75 \%$ work in clinical departments and 90\% had not worked in covid-19 hospitals. (Table 1). The majority (74\%) of participants received information from social media, $59.4 \%$ from seniors and colleagues, $45.6 \%$ from seminars and workshops, and 38.1\% from Radio and television.

\begin{tabular}{|l|l|c|c|}
\hline \multicolumn{4}{|c|}{ Table 1: Demographic data of participating physicians (n=160) } \\
\hline Variable & & $\mathrm{N}$ & $\%$ \\
\hline \multirow{2}{*}{ Gender } & Male & 45 & 28.1 \\
& Female & 115 & 71.9 \\
\hline \multirow{5}{*}{ Age } & $<30$ & 12 & 7.5 \\
& $30-<40$ & 108 & 67.5 \\
& $40-<50$ & 24 & 15 \\
& $\geq 50$ & 16 & 10 \\
\hline \multirow{5}{*}{ Work experience } & $<1$ year & 3 & 1.9 \\
& $1-<3$ years & 6 & 3.8 \\
& $3-<5$ years & 13 & 8.1 \\
\multirow{2}{*}{ Specialty } & $\geq 5$ years & 138 & 86.2 \\
\hline work experience in & Clinical & 142 & 88.75 \\
covid-19 hospital & Academic & 18 & 11.25 \\
\hline
\end{tabular}

The survey revealed that most of participating physicians have good knowledge (98.8\%) with the mean knowledge score (13.02 \pm 1.02$)$ a positive attitude (mean score $9.76 \pm 1.70)$ and good practice (77.5\%) (mean score 5.22 \pm 0.83 ) towards COVID-19 (Table 2). The survey revealed that Limitation of infection control material and Lack 
of policy and Procedures of infection control Practice were the most prevalent Perceived barriers to infection control practice as reported by the participating physicians. Chi-squared analysis revealed that physicians working in clinical departments had greater knowledge than physicians working in academic departments $(p=0.003)$ also physicians with work experience $\geq 5$ years have greater knowledge $(p=0.002)$. Attitude did not differ significantly ( $p>0.05)$ with age, gender, experience or specialty. Regarding practice, physicians aged 30-40 years and physicians working in clinical departments were more likely to follow precautionary practices than those working in academic departments $(p=0.001)$ (table 3). Pearson's correlation tests revealed significant positive linear correlations; knowl-edge/ practice $(r=0.225, p=0.004)$, and attitude/ practice $(r=0.314, p=0.001)$ (Table 4).

\section{Discussion}

This study is one of the earliest studies to determine the knowledge, attitude, and practice towards Covid -19 virus among physicians in Egypt. Regarding demographic characteristics, age of participants in the present study, most of the participants (67\%) were $30-40$ years and $71.9 \%$ of them were females that results coincide with other similar study by Wahed WYA et al conducted in Egypt 2020 where $50.6 \%$ were males. Their mean age was $34.95 \pm 9.32$ years and ranged from 20 to 60 years old. The majority $(60.2 \%)$ of the study participants were working at the university hospital and the others were from general hospitals (25.6\%) or primary health care centers $(14.2 \%)^{(12)}$. Saqlain et al conducted in Pakistan in March 2020 where about $70 \%$ of the participant aged from $30-$ 39 and $50 \%$ of them were females ${ }^{(6)}$. Regarding source of information among participating physicians, in general, most of the participants used social media to obtain information, in this study there was a high prevalence of using social media to obtain information about $70 \%$ of the participants. this was different from another study conducted in Egypt on June 2020, physicians were the most often mentioned as a source of information about COVID-19 followed by the Ministry of health and population (MOHP) or world health organization (WHO) official web sites and social media with only small percent reported courses or training as their source of information $^{(12)}$.

\begin{tabular}{|c|c|c|}
\hline \multicolumn{3}{|c|}{$\begin{array}{l}\text { Table 2: Knowledge, attitude, and practice regarding } \\
\text { COVID-19 among participating physicians }(n=160)\end{array}$} \\
\hline \multirow{3}{*}{ Total knowledge score } & Good & $158(98.8 \%)$ \\
\hline & Poor & $2(1.2 \%)$ \\
\hline & \multicolumn{2}{|c|}{ Mean: $13.02 \pm 1.02$} \\
\hline Total attitude score & \multicolumn{2}{|c|}{ Mean: $9.76 \pm 1.70$} \\
\hline \multirow{3}{*}{ Total practice score } & Good & $124(77.5 \%)$ \\
\hline & Poor & $36(22.5 \%)$ \\
\hline & \multicolumn{2}{|c|}{ Mean: $5.22 \pm 0.83$} \\
\hline
\end{tabular}

Our results were coinciding with the study done by Saqlain et al conducted in Pakistan where 87.68 \% of HCPs used social media as their main source of information ${ }^{(6)}$, similarly study conducted in Vietnam where 91.1\% of the participants used social media 
in gathering their information ${ }^{(13)}$. Participants enrolled in this study showed sufficient knowledge of COVID-19 where $98 \%$ of them have good knowledge. This comes in accordance with study conducted in Egypt where $80.4 \%$ show high level if knowledge, another study in Egypt showed that 91.7\% of physicians have good knowledge. Also similar studies conducted in China, Vietnam, Pakistan show good know-ledge ${ }^{(12,}$ 14). Similarly, an Ugandan study had reported about $70 \%$ of their respondents had sufficient level of knowledge ${ }^{(15)}$, also in an Iranian study it was found that $99 \%$ of respondents had excellent knowledge level regarding the disease modes of transmission but regarding the disease symptoms only $86 \%$ had sufficient knowledge ${ }^{(16)}$. On the other hand, in a study from the United Arab Emirates, poor knowledge about the disease transmission, and the symptom was found in a significant proportion of Health care workers ${ }^{(8)}$. In this study, participants show positive attitude concerning that domain with an overall mean score
9.76 which coincide with an Egyptian study conducted on June 2020 showed an overall positive attitude of Health care workers (HCWs) towards COVID-19 as a preventable disease ${ }^{(12)}$. Similarly a positive attitude with mean score 8.43 was reported ${ }^{(6)}$. Also a similar study conducted in Vietnam showed about $93.3 \%$ a positive attitude towards COVID-19 ${ }^{(13)}$.Participants enrolled in this study show high prevalence of good practice about COVID 19 (77.5\% regarding use of face mask and practice of other precautionary measures). Previously, an Egyptian study showed that $100 \%$ of physicians achieved good practice score ${ }^{(14)}$. These results were coincident with Saqlain et al study where $88.7 \%$ of the participants show good practice regarding COVID-19 ${ }^{(6)}$. This study revealed that Limitation of infection control material is about $88.75 \%$ and Lack of policy and Procedures of infection control Practice is $84.38 \%$ which were the most prevalent Perceived barriers to infection control practice as reported by the participating physicians..

\begin{tabular}{|c|c|c|c|c|c|c|c|c|c|c|c|}
\hline \multicolumn{8}{|c|}{ Table (3): Difference in physician's Knowledge, attitude, and practice regarding COVID-19 } \\
by demographics (n=160)
\end{tabular}

*Significant at 0.05 level (two-tailed). $\chi^{2}$ - Chi-squared. $\mathrm{f}$ - Fisher's Exact. $\mathrm{t}$ - Independent samples t-test. A - ANOVA (F-test). 
Similarly in a study conducted in Egypt on June 2020 lack of the personal protective equipment was the most commonly mentioned cause for feeling more susceptible followed by dealing with the public who are not committed to preventive measures together with ill ventilation and over crowdedness in the workplace ${ }^{(12)}$. Saqlain et al found that Overcrowding in emergency rooms was perceived as a barrier to infection control by the majority about $52.9 \%$ of $\mathrm{HCWs}^{(6)}$. These findings are important and should be addressed by the government and policy makers to establish effective policies focusing on the previously barriers to infection control and, ultimately, spread of disease. This study revealed that physicians working in clinical departments had greater knowledge than physicians working in academic departments $(p=0.003)$, Also physicians with work experience $\geq 5$ years have greater knowledge $(p=0.002)$.

\begin{tabular}{|l|c|c|}
\hline \multicolumn{3}{|c|}{$\begin{array}{c}\text { Table 4: Correlation between scores of knowledges, attitude, } \\
\text { and practice regarding COVID-19 }\end{array}$} \\
\hline Variable & Correlation Coefficient & p-Value \\
\hline Knowledge-Attitude & 0.137 & 0.084 \\
\hline Attitude-Practice & 0.314 & $\mathbf{0 . 0 0 1}^{*}$ \\
\hline Knowledge-Practice & 0.225 & $\mathbf{0 . 0 0 4}$ \\
\hline * Statistically significant at 0.05 level (2 tailed). \\
\hline
\end{tabular}

Similarly, Saqlain et al study revealed that there is no difference in mean attitude score was found by experience ( $p>0.05)$ of 414 participants where $88.7 \%(n=367)$ had positive practice (score $=5-6$ ) in following precautions to avoid COVID-19. Also revealed that majority $(95.7 \%, n=112)$ of HCWs having greater experience ( $>5$ years) had good practice of following precautions compared to less experienced counterparts $(p=0.020)^{(6)}$. In this study, Attitude did not differ significantly $(p>0.05)$ with age, gender, experience, or specialty. In contrast to, Saqlain et al., other studies demonstrate that age was significantly associated with good knowledge as $95.5 \%$ $(n=284)$. HCPs of age $<30$ years had good knowledge compared to HCWs of other age groups ${ }^{(6)}$. Also in an Egyptian study, Knowledge level about COVID-19 was significantly associated with younger age groups especially 20-30 years. This could be explained by the fact that younger persons tend to use the internet more frequently. In Egypt, Age groups between 18 and 40 years, represent more than $75 \%$ of Facebook users ${ }^{(12)}$. This study revealed a positive correlation between know-ledge and attitude, in coincidence with study conducted in Egypt ${ }^{(12)}$. Similarly, a in Chinese study revealed significant positive linear correlations between knowledge and attitude, knowledge and practice as well as attitude and practice ${ }^{(6)}$.

\section{Conclusion}

There is good knowledge regarding COVID19 among physicians. Appropriate practice correlates with better knowledge and positive attitude towards COVID-19 infection. More training programs on protective measures to improve attitude among physicians and healthcare workers are necessary to win the fight against COVID-19.

\section{Study limitations}

This study was an online survey; thus responses might be subject to recall bias. The 
generalizability of the study may have been limited by the sampling method as we used a convenient sample.

Funding: None

Conflicts of interest: None.

Acknowledgment: The authors would like to thank the participants for their co-operation to accomplish this work.

Authors' contributions: All authors were equally contributing to this work.

\section{References}

1. Schoeman D, and Fielding BC. Coronavirus envelope protein: current knowledge. Virol J. 2019; 16 (1):1-22.

2. Yang $X, Y u Y, X u$ J, et al. Clinical course and outcomes of critically ill patients with SARS-CoV-2 pneumo-nia in Wuhan, China: a single-centered, retrospective, observa-tional study. The Lancet Respiratory Medicine. 2020.

3. Guan $\mathrm{W}-\mathrm{j}, \mathrm{Ni} Z-\mathrm{y}, \mathrm{Hu} \mathrm{Y}$, et al. Clinical characteristics of coronavirus dis-ease 2019 in China. N Engl J Med. 2020; 382 (18):1708-20.

4. Mehta P, McAuley DF, Brown M, et al. COVID-19: consider cytokine storm syndromes and immuno-suppression. Lancet (London, England). 2020; 395 (10229): 1033.

5. Tao Y, Yang R, Wen C, et al. SARS-CoV-2 entry related genes are comparably expressed in children's lung as adults. medRxiv. 2020.

6. Saqlain M, Munir MM, ur Rehman S, et al. Knowledge, attitude, practice and perceived barriers among healthcare professionals regarding COVID-19: A Cross-sectional survey from Pakistan. The Journal of Hospital Infection. 2020.

7. Lei S, Jiang F, Su W, et al. Clinical characteristics and outcomes of patients undergoing surgeries during the incubation period of COVID-19 infection. E Clinical Medicine. 2020:100331.
8. Bhagavathula AS, Aldhaleei WA, Rahmani J, et al. Novel coronavirus (COVID-19) knowledge and perceptions: a survey on healthcare workers. MedRxiv. 2020.

9. Gan WH, Lim JW, David K. Preventing intra-hospital infection and transmission of COVID-19 in healthcare workers. Safety and Health at Work. 2020.

10. Nemati $M$, Ebrahimi B, Nemati $F$. Assessment of Iranian nurses' knowledge and anxiety toward COVID19 during the current outbreak in Iran. Arch Clin Infect Dis. 2020; 15(COVID-19): e102848.

Omrani A, and Shalhoub S. Middle East respiratory syndrome corona-virus (MERS-CoV): what lessons can we learn? J Hosp Infect. 2015; 91 (3):188-96.

11. Wahed WYA, Hefzy EM, Ahmed MI, et al. Assessment of knowledge, attitudes, and percep-tion of health care workers regarding COVID-19, a cross-sectional study from Egypt. J Community Health. 2020; 45 (6): 1242-51.

12. Huynh G, Nguyen TNH, Vo KN, et al. Knowledge and attitude toward COVID19 among healthcare workers at District 2 Hospital, Ho Chi Minh City. Asian Pac J Trop Med. 2020;13(6):260.

13. Mohammed ASE. knowledge, Attitudes, and Practices towards COVID-19 among Health Care Workers in Primary Health Care Units Dar El Salam, Suhag, Egypt. SMJ . 2021; 25(1):50-8.

14. Olum R, Chekwech G, Wekha G. Coronavirus disease-2019: know-ledge, attitude, and practices of health care workers at Makerere University Teaching Hospitals, Uganda. Frontiers in public health. 2020; 8:181. 